\title{
Lattice QCD and Nuclear Physics
}

\section{Computations of Hadron-Hadron scattering}

\author{
Konstantinos Orginos ${ }^{12}$ \\ 1 Department of Physics, College of William and Mary, Williamsburg, VA 23187-8795 \\ 2 Jefferson Laboratory, 12000 Jefferson Avenue, Newport News, VA 23606
}

Received: date / Revised version: date

\begin{abstract}
A steady stream of developments in Lattice QCD have made it possible today to begin to address the question of how nuclear physics emerges from the underlying theory of strong interactions. Central role in this understanding play both the effective field theory description of nuclear forces and the ability to perform accurate non-perturbative calculations in low energy QCD. Here I present some recent results that attempt to extract important low energy constants of the effective field theory of nuclear forces from lattice QCD.
\end{abstract}

PACS. 11.15.Ha Lattice gauge theory - 12.38.Gc Lattice QCD calculations - 12.38.Aw General properties of QCD - 12.38.-t Quantum chromodynamics - 21.30.-x Nuclear forces - 13.75.Cs Nucleon-nucleon interactions

\section{Introduction}

Computing from first principles the properties of hadron interactions that lead to the formation of atomic nuclei is a major challenge for lattice QCD. The fact that the QCD dynamics is at scales of $1 \mathrm{GeV}$ while the nuclear forces are resulting effects of the order a few $\mathrm{MeV}$ creates a classic two scale problem. Effective field theory techniques are a powerful tool to tackle such problems. The matching though of the effective field theory to the high energy scale theory, namely QCD, requires non-perturbative calculations within QCD. Lattice QCD is the only way to perform such calculations.

For realistic lattice calculations dynamical fermions with pion masses below $400 \mathrm{MeV}$ are needed. This allows chiral effective field theories to be used with some reliability. In addition a dynamical strange quark is required in order to guarantee that the low energy constants of the Chiral Lagrangian match those of the physical theory. Large physical volumes are also needed so that finite volume systematic errors are under control. Although this task seems formidable, in the last several years there were developments in Lattice QCD calculations that permit the performance of phenomenologically interesting calculations that address these questions.

The emergence of fermions that respect chiral symmetry [1-4] on the lattice was one of the major recent developments in Lattice QCD. These formulations of lattice fermions allow us to reduce the lattice spacing errors and approach the continuum limit in a smoother manner. In addition, the development of improved Kogut-Susskind fermion actions $[5,6]$ that significantly reduce the $O\left(a^{2}\right)$ errors, allowed for cheap inclusion of quark loop effects in the QCD correlation functions computed on the lattice. With this formulation we can work at volumes as large as $3.5 \mathrm{fm}$ and quark masses as low as $1 / 10$ th of the strange quark mass.

In the work I present here, we used domain wall fermions for the valence sector and Kogut-Susskind fermions to represent the quark loops. This mixed action calculation allows us to both take advantage of the chiral symmetry properties of domain wall fermions and have quark loops with masses close to the physical regime. Although one might think that this mixed action scheme is a complication difficult to control, in practice it has been shown that the effects of the miss-match between the sea and the valence sectors are small in the case of flavor non-singlet quantities. In theory all these complications can be taken care of in the context of mixed action chiral perturbation theory $[7,8]$. Another problem with Kogut-Susskind fermions is that there is still a theoretical issue of the validity of computations with number of flavors not an integer multiple of four. However, recent theoretical work indicates that the troublesome non-localities of the lattice action are going away in the continuum limit [?,10-12]. Hence these effects are most likely taken care of in the continuum limit.

Given the available technology for lattice QCD calculations, there is a variety of physical observables with direct impact to nuclear physics that can be computed. The nucleon mass spectrum, decay constants and axial couplings are now standard lattice calculations that can be done with very good precision with reliable control of the sys- 


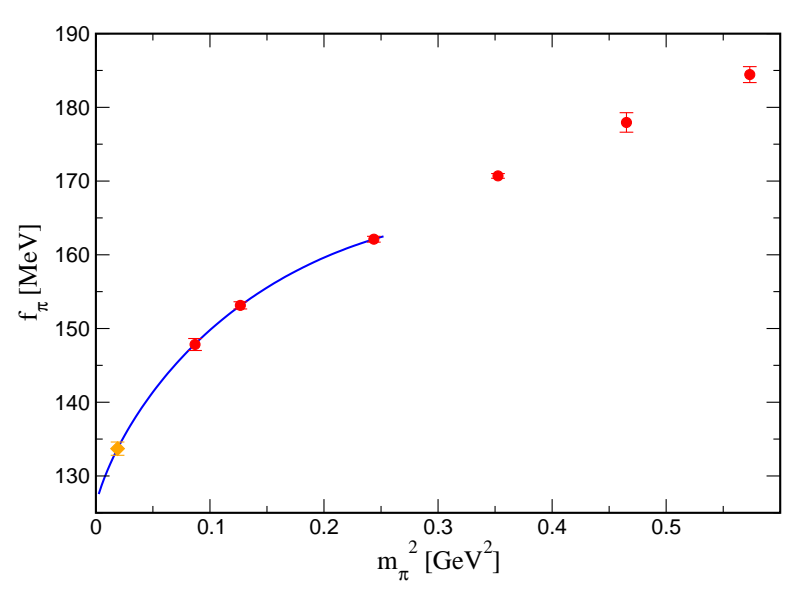

Fig. 1. The pion decay constant. Only the lighter three points are used in the fit. The diamond is the extrapolated value with its statistical error.

tematic errors involved. In addition to these types of calculations, more recently lattice QCD calculations of scattering lengths and phase shifts have began to emerge [13-15] In the following I am presenting a few of such calculations that I have worked on with my collaborators in LHPC and NPLQCD.

\section{Two particles in a box}

Although the energy levels of single particle states can be easily computed from Euclidean two point correlation functions, scattering amplitudes cannot be extracted from Euclidean four point functions in infinite volume except on kinematic thresholds, as stated by the Maiani-Testa nogo theorem. However, Lüscher showed $[16,17]$ that the swave scattering amplitude for two particles below inelastic thresholds can be determined using the measurements of one or more energy levels of the two-particle system in a finite volume. In particular, he showed that in the center of mass frame, the s-wave energy levels of two particles of identical mass $m$ in a finite volume are shifted from those of two non-interacting particles by an amount that is related to the scattering amplitude. This energy level shift is $\Delta E_{n} \equiv E_{n}-2 m=2 \sqrt{p_{n}^{2}+m^{2}}-2 m$ where $p_{n}$ is defined by this equation and satisfies

$$
p_{n} \cot \delta\left(p_{n}\right)=\frac{1}{\pi L} \mathbf{S}\left(\frac{p_{n}^{2} L^{2}}{4 \pi^{2}}\right)=\frac{1}{a}+\frac{1}{2} r p_{n}^{2}+\cdots
$$

where $\delta\left(p_{n}\right)$ is the elastic-scattering phase shift, $a$ and $r$ are the scattering length and effective range respectively, $L$ is the length of the spatial dimension in a cubicallysymmetric lattice, and

$$
\mathbf{S}(\eta) \equiv \lim _{\Lambda \rightarrow \infty}\left[\sum_{\mathbf{j}}^{|\mathbf{j}|<\Lambda} \frac{1}{|\mathbf{j}|^{2}-\eta}-4 \pi \Lambda\right]
$$

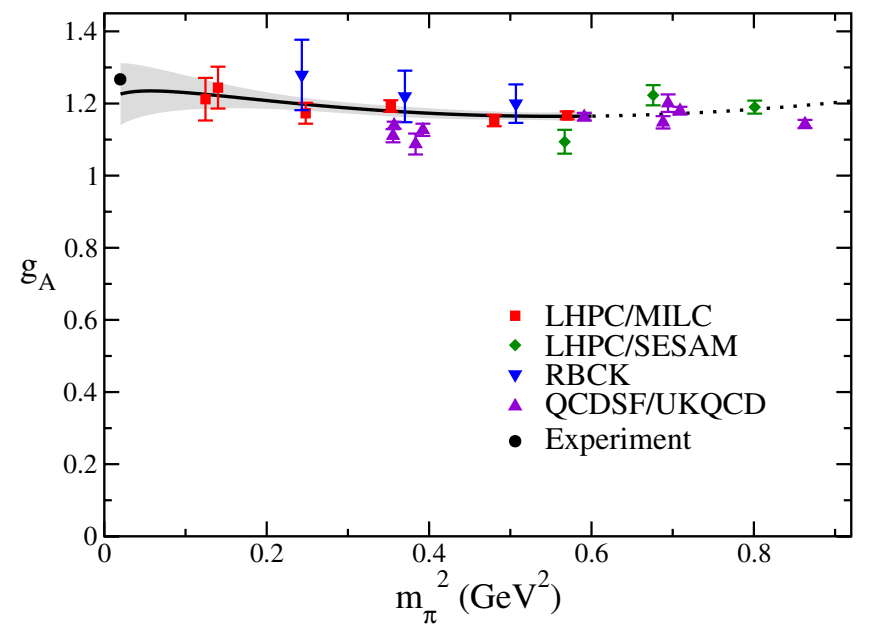

Fig. 2. The nucleon axial coupling.

This definition is equivalent to the analytic continuation of zeta-functions presented by Lüscher [18].

\section{The Lattice calculation}

In our computation we use the mixed-action scheme developed by LHPC $[19,20]$ using domain-wall valence quarks on $N_{f}=2+1$ asqtad-improved [5,6] MILC configurations generated with rooted ${ }^{1}$ staggered sea quarks [21]. For more details on the numerical calculations see [19, $20,15,14]$. The essential feature of our calculation is that the pion masses ranged between $300 \mathrm{MeV}$ and $770 \mathrm{MeV}$ and that the strange quark mass was fixed near its physical mass. The domain wall fermion mass was tuned so that the domain wall pions matched the Kogut-Susskind Goldston pion mass. The scattering length is extracted by computing on the lattice the energy level shift $\Delta E_{0}$. In order to do this, the ratio of correlators of the two particle state to the product of the single particle correlators is fitted to a single exponential $[15,14]$. The decay rate of this exponential is the energy level shift we need.

\subsection{Decay Constants}

The pseudoscalar decay constants are important parameters of the Chiral Lagrangian which we would like to compute from lattice QCD. These constants have been computed lately to great precision by MILC collaboration [22, $23]$ using staggered fermions. The dominant error ( $3 \%)$ in these calculations is systematic (due to scale setting, chiral extrapolations, continuum limit) rather than statistical. Within all the errors the results are in remarkable agreement with experiment. We have repeated these calculations using the mixed action scheme described above.

\footnotetext{
1 The "legality" of the rooting procedure has been questioned and investigated in recent lattice literature. Here due to space limitations I cannot address the issues raised fully.
} 

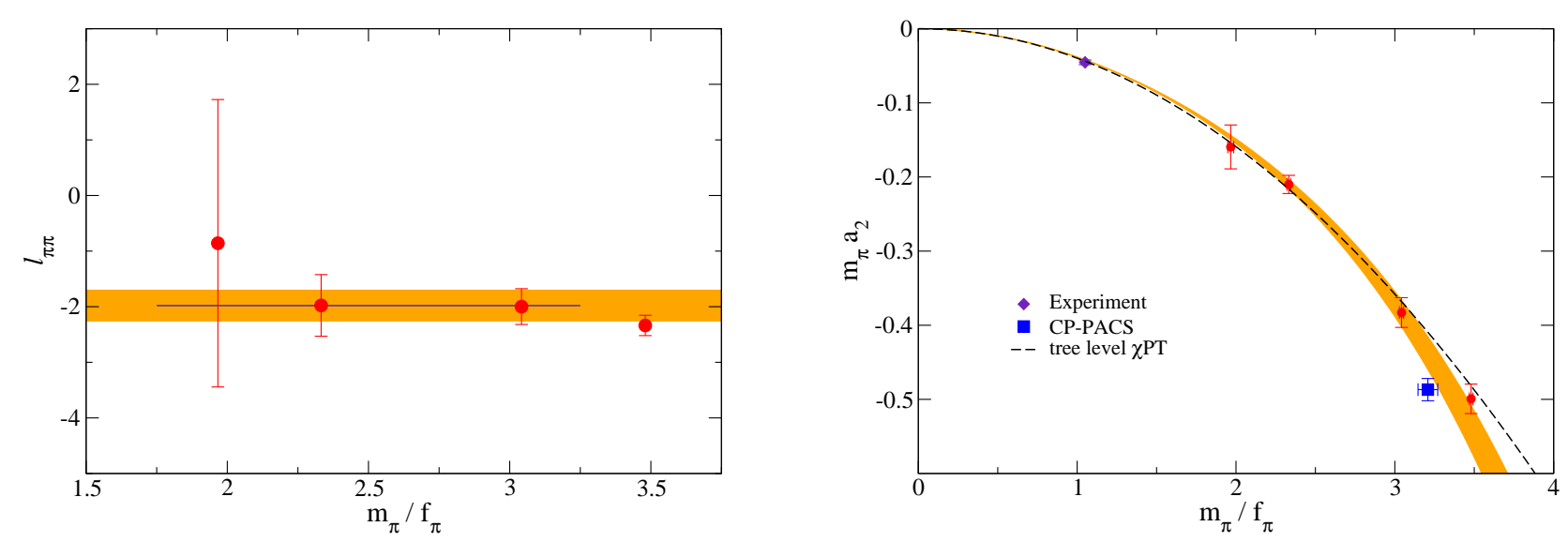

Fig. 3. The $\mathrm{I}=2 \pi-\pi$ scattering length. The fit to the lattice results to extract the only fitted parameter $l_{\pi \pi}$ (left). The resulting fit togethe $\mathrm{r}$ with the tree level result and the lightest CP-PACS point (right).

Our results for the ratio of the kaon to pion decay constant ratio are presented in detail in [24]. We find that $f_{K} / f_{\pi}=1.218(2)_{-0.024}^{+0.011}$ where the first error is statistical and the second is systematic due to the chiral extrapolation. In addition, by fitting to leading order chiral perturbation theory formulas [25] we obtain $f_{\pi}=133.7(9)(3.0)$ $\mathrm{MeV}$, where the second error is systematic due to the chiral fit. The fit shown in Fig. 1 results in a low energy constant $\bar{l}_{4}^{\text {phys }}=4.39(3)$ compatible with the experimental expectations and the lower statistics result obtained in [15]. The lower four points plotted in Fig. 1 are high statistics NPLQCD data while the rest are LHPC data. One remarkable feature of this plot is the fact that for pion masses above $400 \mathrm{MeV}$ the data are almost linear while below there is a clear indication of the chiral log curvature.

Although in principle one needs to use the effective theory that takes into account the taste breaking effects in the sea sector [7], in practice it turns out that the regular continuum chiral perturbation theory formulas fit our data quite well, leading us to believe that the taste breaking effects on the decay constant are rather small.

The compatibility of our results with those obtained by MILC collaboration is very encouraging for the the mixed action scheme we have adopted.

\subsection{Nucleon axial coupling}

An other low energy constant useful for the effective field theories needed to describe the nuclear forces is the nucleon axial coupling. The ability to reproduce the well known experimental result has been a challenge for lattice QCD. Recently though, the use of light pion masses together with the understanding of the finite volume corrections [26-28] has led to remarkable improvement in the lattice results. In Fig. 2 the results by LHPC presented in [27] are shown. Our chiral extrapolation using the fi- nite volume formulas of [29] obtain a value of $g_{A}=1.22(8)$ which is in agreement with the experimental result. This result still has unknown systematic errors due to continuum and chiral extrapolation, but we expect both these errors to be smaller, or at least comparable to our statistical error.

Recent work by Bernard and Meissner [30], going to two loops chiral perturbation theory, indicates that one may need pion masses below $300 \mathrm{MeV}$ before, reliable chiral extrapolations can be obtained. Certainly, the lattice results shown here, indicate that improving the control of the chiral extrapolation would definitely benefit from lower masses. In fact it seems that this is the only way one can improve the the precision on the $g_{A}$ calculation both from the point of view of statistical and systematic errors. LHPC is currently working in pushing closer to the chiral limit. Using the Kogut-Susskind lattices produced by MILC it is expected to be able to perform calculations in the range of $250 \mathrm{MeV}$ pions. In addition, calculations on a smaller lattice spacing is in the pipe line in order to control the continuum extrapolation error.

\subsection{Charge symmetry breaking}

Recently [31], using partially quenched chiral perturbation theory, we have calculated the proton-neutron mass splitting due to charge symmetry breaking. Although this splitting is small one can obtain an accurate estimate in lattice calculations taking advantage of the statitsitcal correlations between quantities computed on the same ensemble. The neutron-proton mass difference due to the up-down quark mass splitting $\left(m_{u}-m_{d}\right)$ is related to this splitting by

$$
M_{n}-\left.M_{p}\right|^{d-u}=\frac{2}{3}(2 \bar{\alpha}-\bar{\beta})\left(\frac{1-\eta}{1+\eta}\right) m_{\pi}^{2},
$$



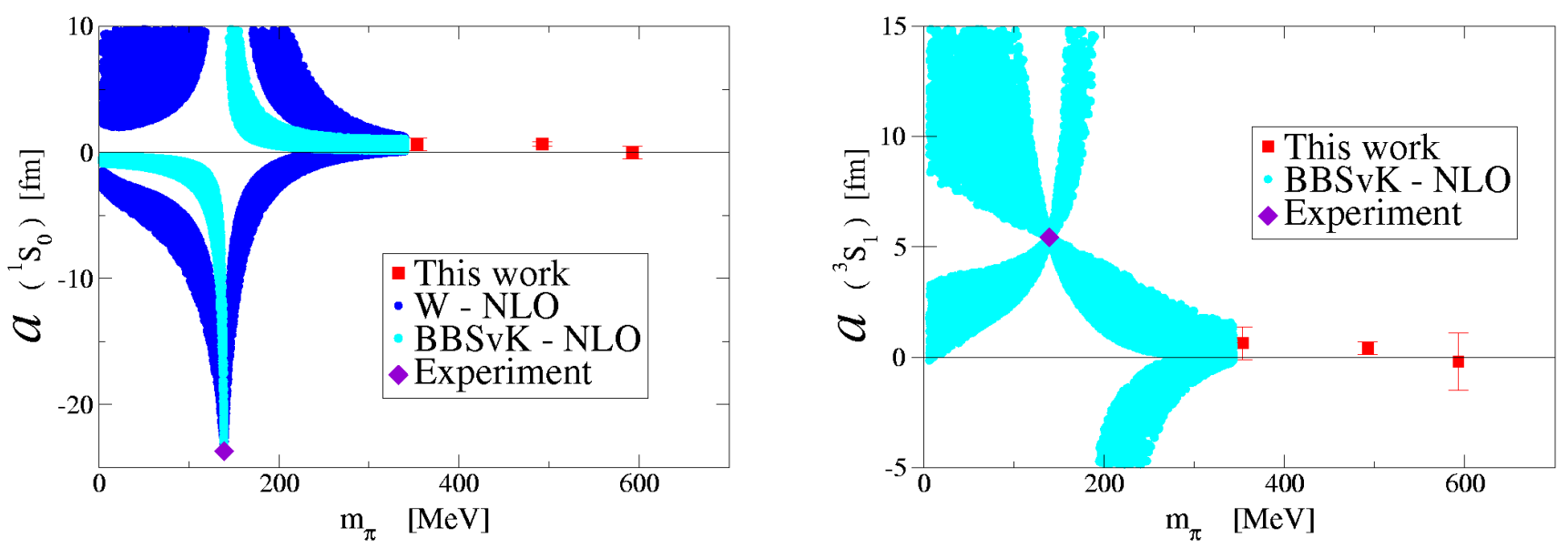

Fig. 4. The nucleon-nucleon scattering lengths using the opposite sign convention from the $\pi-\pi$ case.

where $\eta=m_{u} / m_{d}$ and $2 \bar{\alpha}-\bar{\beta}$ is the strong iso-spin breaking term in the chiral expansion of the proton (neutron) mass. Partially quenched calculations with non-degenerate up and down quarks, ie. keeping the sea quarks degenerate, allow us to compute from lattice calculations the term $2 \bar{\alpha}-\bar{\beta}$ to great precision. Using $\eta=0.43(1)(8)$ determined by MILC [23] we can obtain

$$
M_{n}-\left.M_{p}\right|^{d-u}\left(m_{\pi}^{p h y s}=2.26(57)(42) M e V\right.
$$

using the $O\left(m_{q}^{3 / 2}\right.$ partially quenched formulas. This is to be compared to the the experimental value of

$$
M_{n}-\left.M_{p}\right|_{e x p} ^{d-u}=2.05(30) M e V
$$

after correcting for electromagnetic effects [32].

\subsection{Pion scattering}

The scattering length is the observable that is directly related to the hadron-hadron interactions. Using Luscher's finite volume technique described above we computed the pion - pion scattering length in the $\mathrm{I}=2$ channel. Our results are plotted in Figure 3 together with the lightest CP-PACS point [13]. This calculation is the first dynamical fermion calculation at these light pion masses. The lattice results are fitted using the one loop chiral perturbation formula [25]

$$
m_{\pi} a_{2}=-\frac{m_{\pi}^{2}}{8 \pi f_{\pi}^{2}}\left[1+\frac{3 m_{\pi}^{2}}{16 \pi^{2} f_{\pi}^{2}}\left(\log \frac{m_{\pi}^{2}}{f_{\pi}^{2}}+l_{\pi \pi}\left(f_{\pi}\right)\right)\right]
$$

where $l_{\pi \pi}\left(f_{\pi}\right)$ is the Gasser-Leutwyler low energy constant which scales as

$$
l_{\pi \pi}(\mu)=l_{\pi \pi}\left(f_{\pi}\right)+2 \log \left(\mu / f_{\pi}\right) .
$$

The fitted result at the physical point is

$$
m_{\pi} a_{2}=-0.0422(3)(18)
$$

where the first error is statistical and the second is an estimated systematic error due to chiral extrapolation. This result is slightly different from the one in [15] due to increased statistics. After scaling to $1 \mathrm{GeV}$ using eq. (7), the low energy constant $l_{\pi \pi}$ is $l_{\pi \pi}(1 \mathrm{GeV})=2.1(3)$. In the above fits only the lightest three points were used. This is in good agreement with the best experimental result

$$
m_{\pi} a_{2}=-0.0454(31) .
$$

Mixed action $\chi$-PT [8] has insignificant effect on the final result. The authors of [8] estimated it to be -0.003 . We are currently extending our studies of meson-meson scattering to the kaon-pion [33] and kaon-kaon systems.

\subsection{Nucleon-Nucleon scattering}

We also computed the scattering length in the nucleonnucleon system where very little lattice work has been done $[34,35]$. Our results are plotted in Figure 4. The shaded areas are the allowed regions that various effective field theory approaches predict for these scattering lengths, if we use the experimental points together with the lightest lattice point to constrain their parameters [36$41,14]$. Clearly, the lightest lattice point is on the boundary of applicability of these effective field theories, indicating that we need significantly lighter pion masses in order to be able to predict from QCD the physical scattering lengths in these processes. In addition to lighter pion masses, we need to study the volume dependence of the energy levels in order establish the scattering nature of the states we observe. Certainly, this calculation is far from definitive. It is an attempt to understand what it takes to perform such calculations. In the next few years we hope to improve substantially the quality of our results. Nonetheless, even at such an early stage, our calculation undoubtedly shows this very valuable piece of information: in the range of pion masses used here, the scattering lengths in the nucleon-nucleon channel are very different from their values at the physical point. 
We are currently working on significantly enhancing our statistics in order to be able to reduce our error bars and obtain unambiguous signals for the scattering lengths. Also we are investigating techniques to enhance our signal and alternative lattice formulations that will allow us to push towards the chiral limit given the computational resource available to us.

\section{Conclusions}

In this talk I have presented a number of lattice calculations that are relevant to nuclear physics. The ultimate goal is to obtain an understanding of nuclear forces from QCD. Together with effective field theory techniques, lattice QCD is valuable tool in this endeavor. It is the only way to compute from QCD the low energy constants needed by the low energy effective field theories. Recent theoretical, algorithmic and hardware developments have made it possible today to perform phenomenologically interesting calculations with direct impact in our understanding of the nature and phenomenology of strong interactions. Calculations directly related to the nucleonnucleon interactions are just starting indicating that within then next few years one can expect significant results in this area.

\section{Acknowledgments}

I would like to thank my collaborators in NPLQCD, and LHPC. In particular I would like to acknowledge Silas Beane, Paulo Bedaque, and Martin Savage for their contributions in every aspect of the calculations done within NPLQCD. All calculations were done with the QDP++/ Chroma programming environment [42] at the JLab LQCD cluster. This work was supported in part by DOE contract DE-AC05-84ER40150, under which SURA operated the Thomas Jefferson National Accelerator Facility (JLab) and in part by DOE contract DE-AC05-06OR23177 under which Jefferson Science Associates, LLC currently operates JLab.

\section{References}

1. D.B. Kaplan, Phys. Lett. B288, 342 (1992), [http://arXiv.org/abs]hep-lat/9206013

2. R. Narayanan, H. Neuberger, Nucl. Phys. B412, 574 (1994), [http://arXiv.org/abs]hep-lat/9307006

3. V. Furman, Y. Shamir, Nucl. Phys. B439, 54 (1995), [http://arXiv.org/abs]hep-lat/9405004

4. H. Neuberger, Phys. Lett. B417, 141 (1998), hep-lat/9707022

5. K. Orginos, D. Toussaint, R.L. Sugar (MILC), Phys. Rev. D60, 054503 (1999), hep-lat/9903032

6. K. Orginos, D. Toussaint (MILC), Phys. Rev. D59, 014501 (1999), [http://arXiv.org/abs]hep-lat/9805009

7. O. Bar, C. Bernard, G. Rupak, N. Shoresh, Phys. Rev. D72, 054502 (2005), hep-lat/0503009
8. J.W. Chen, D. O'Connell, R.S. Van de Water, A. WalkerLoud, Phys. Rev. D73, 074510 (2006), hep-lat/0510024

9. S.R. Sharpe, Proc. Sci. LAT2006 (2006), hep-lat/0610094

10. Y. Shamir (2006), hep-lat/0607007

11. C. Bernard, M. Golterman, Y. Shamir, Phys. Rev. D73, 114511 (2006), hep-lat/0604017

12. C. Bernard, Phys. Rev. D73, 114503 (2006), hep-lat/0603011

13. T. Yamazaki et al. (CP-PACS), Phys. Rev. D70, 074513 (2004), hep-lat/0402025

14. S.R. Beane, P.F. Bedaque, K. Orginos, M.J. Savage, heplat/0602010 (2006), hep-lat/0602010

15. S.R. Beane, P.F. Bedaque, K. Orginos, M.J. Savage (NPLQCD), Phys. Rev. D73, 054503 (2006), hep-lat/0506013

16. M. Luscher, Commun. Math. Phys. 104, 177 (1986)

17. M. Luscher, Commun. Math. Phys. 105, 153 (1986)

18. S.R. Beane, P.F. Bedaque, A. Parreno, M.J. Savage, Phys. Lett. B585, 106 (2004), hep-lat/0312004

19. D.B. Renner et al. (LHP), Nucl. Phys. Proc. Suppl. 140, 255 (2005), hep-lat/0409130

20. R.G. Edwards et al. (LHPC), Proc. Sci. LAT2005, 056 (2005), hep-lat/0509185

21. C.W. Bernard et al., Phys. Rev. D64, 054506 (2001), [http://arXiv.org/abs]hep-lat/0104002

22. C. Aubin, C. Bernard, Phys. Rev. D68, 074011 (2003), hep-lat/0306026

23. C. Aubin et al. (MILC), Phys. Rev. D70, 114501 (2004), hep-lat/0407028

24. S.R. Beane, P.F. Bedaque, K. Orginos, M.J. Savage (2006), hep-lat/0606023

25. J. Gasser, H. Leutwyler, Ann. Phys. 158, 142 (1984)

26. S. Sasaki, K. Orginos, S. Ohta, T. Blum (the RIKENBNL-Columbia-KEK), Phys. Rev. D68, 054509 (2003), hep-lat/0306007

27. R.G. Edwards et al. (LHPC), Phys. Rev. Lett. 96, 052001 (2006), hep-lat/0510062

28. A.A. Khan et al. (2006), hep-lat/0603028

29. S.R. Beane, M.J. Savage, Phys. Rev. D70, 074029 (2004), hep-ph/0404131

30. V. Bernard, U.G. Meissner (2006), hep-lat/0605010

31. S.R. Beane, K. Orginos, M.J. Savage (2006), hep-lat/0605014

32. J. Gasser, H. Leutwyler, Phys. Rept. 87, 77 (1982)

33. S.R. Beane et al. (2006), hep-lat/0607036

34. M. Fukugita, Y. Kuramashi, H. Mino, M. Okawa, A. Ukawa, Phys. Rev. Lett. 73, 2176 (1994), hep-lat/9407012

35. M. Fukugita, Y. Kuramashi, M. Okawa, H. Mino, A. Ukawa, Phys. Rev. D52, 3003 (1995), hep-lat/9501024

36. S. Weinberg, Phys. Lett. B251, 288 (1990)

37. S. Weinberg, Nucl. Phys. B363, 3 (1991)

38. C. Ordonez, L. Ray, U. van Kolck, Phys. Rev. C53, 2086 (1996), hep-ph/9511380

39. D.B. Kaplan, M.J. Savage, M.B. Wise, Nucl. Phys. B534, 329 (1998), nucl-th/9802075

40. D.B. Kaplan, M.J. Savage, M.B. Wise, Phys. Lett. B424, 390 (1998), nucl-th/9801034

41. S.R. Beane, P.F. Bedaque, M.J. Savage, U. van Kolck, Nucl. Phys. A700, 377 (2002), nucl-th/0104030

42. R.G. Edwards, B. Joo (SciDAC), Nucl. Phys. Proc. Suppl. 140, 832 (2005), hep-lat/0409003 